\title{
Real-Time High-Sensitivity Impedance Measurement Interface for Tethered BLM Biosensor Arrays
}

\author{
Yuksel Temiz, Frank K. Gurkaynak, \\ Samuel Terrettaz, Horst Vogel, \\ Giovanni De Micheli, Yusuf Leblebici \\ Swiss Federal Institute of Technology (EPFL) \\ Lausanne, Switzerland \\ Email: yuksel.temiz@epfl.ch
}

\author{
Carlotta Guiducci, Luca Benini \\ University of Bologna \\ Bologna, Italy \\ Email: carlotta.guiducci@unibo.it
}

\begin{abstract}
This paper presents a switched-capacitor (SC) current integrator circuit for impedance measurement of tethered bilayer lipid membrane (tBLM) biosensors. The circuit comprises a small number of high performance components enabling enhanced experimental flexibility and reliability. The sensitivity is improved significantly by suppressing the output offset through pseudo-differential operation, using $\mathrm{R}-\mathrm{C}$ components for the reference impedance. The sensing and reference electrodes are excited with low-amplitude differential voltage pulses and the current response to membrane resistance $\left(R_{M}\right)$ change of the tBLM biosensor is converted to voltage by a precision, low-noise $\mathrm{SC}$ integrator available as a single-package IC. Tests with both electrical models and actual biosensors demonstrated that the proposed circuit operates with high sensitivity and can be used in single chip versions for low-cost and high-sensitive tBLM biosensor arrays, featuring multiple electrode sites.
\end{abstract}

\section{INTRODUCTION}

Measuring ion channel activity is of central importance in many bioanalytical and medical applications including the biophysical characterization of channels, drug screening or medical point-of-care diagnostics to mention a few. As an example of ever increasing relevance in this context we consider biosensors based on ion channels, where the measurement of electrical impedance modulation is the key element for detecting and quantifying specific biological functions, such as selective binding of a ligand to a cognate receptor. Classical electrochemical impedance spectroscopy (EIS) techniques, like lock-in amplifier measurements, allow quantification of channel activity [1]. However, these techniques require high-cost, heavy laboratory equipments, which make them inconvenient for low-cost, low-power, and miniaturized lab-on-a-chip systems composed of dense arrays of biosensors. Such miniaturized systems can only be realized through dedicated electronics capable of measuring very small electrical changes in the sensor. Additionally, monitoring the real-time transient sensor response can give further important information on the ion channel properties.
Here, we provide an IC-compatible approach to fulfill the requirements for highly-sensitive and real-time impedance measurements of tBLM biosensor arrays.

\section{TETHERED BLM BIOSENSOR}

tBLMs are ideally suited for reconstituting membrane proteins, in a functionally active and mechanically stable form to be investigated by surface sensitive analytical techniques. The variation of the electrical resistance of tBLMs containing ion channels by ligand binding has been used in biosensors [1-6]. Attractive features of this type of biosensors include high amplification of ligand binding by ligand-gated ion channel currents (up to $10^{8}$ ions/sec), high specificity and biocompatibility. A schematic view of a tBLM on a gold electrode is shown in Fig.1. The lipid bilayer is attached to the gold surface via sulfur-terminated hydrophilic spacers of thiolipids [1]. The resulting aqueous phase between the electrode surface and the lipid bilayer is designed to accommodate the extramembraneous parts of membrane proteins. Tethered lipid bilayers can be formed quickly and easily by self-assembly; they show an exceptionally high mechanical stability $[3,5]$.

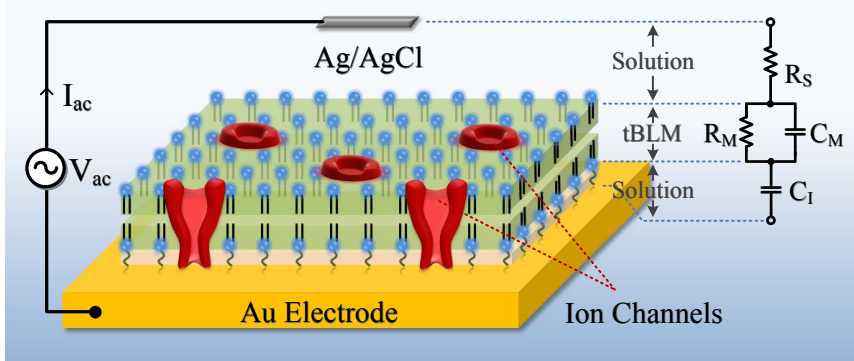

Figure 1. Schematic view and equivalent model of a two-electrode electrochemical cell with an $\mathrm{Ag} / \mathrm{AgCl}$ counter electrode and a gold working electrode coated with a tBLM. The impedance of the large $\left(>1 \mathrm{~cm}^{2}\right.$ effective area) counter electrode is negligible when the electrolyte solution comprises enough chloride ions (usually $\geq 0.1 \mathrm{M}$ ).
This work is supported in part by EPFL Integrated Systems Centre (Centre SI). 
Fig.1 also illustrates the simplified electrical equivalent model composed of capacitors and resistors. More complicated sensor models employing higher order lumped components and non-ideal circuit elements, such as constant phase element, have been proposed [4]. In the simplified circuit model, $\mathrm{R}_{M}$ and $\mathrm{C}_{\mathrm{M}}$ represent the resistance and capacitance of the lipid bilayer membrane, respectively, while $R_{S}$ is the resistance of the solution and $C_{I}$ describes the interfacial capacitance.

Fig.2 shows the magnitude and phase parts of a typical impedance spectrum of tBLM biosensors. This plot implies that $\mathrm{C}_{\mathrm{I}}$ dominates the response at very low frequencies, whereas $C_{M}$ becomes effective at relatively high frequencies. At a mid-frequency range, generally between $1 \mathrm{~Hz}$ and $100 \mathrm{~Hz}, \mathrm{R}_{\mathrm{M}}$ is the element dominating the impedance response. Since modulation of the $R_{M}$ is used to detect or quantify the ligand binding to an ion channel, this frequency range is quite important for attaining maximal sensitivity of the biosensor.

\section{READOUT CIRCUIT}

In tBLM sensors, changes in the membrane resistance $\left(\Delta \mathrm{R}_{\mathrm{M}}\right)$ cannot be detected by $\mathrm{DC}$ measurement techniques due to the DC-blocking effect of the $\mathrm{C}_{\mathrm{I}}$. Thus, the sensor has to be excited with a time-varying current or voltage signal whose amplitude and frequency should be adjusted carefully to avoid excessive signal amplitudes or large DC bias voltages which may easily cause permanent damage of the tBLM. Here, a low-amplitude (below $50 \mathrm{mV}_{\mathrm{pp}}$ ) and zero-offset voltage pulse at the frequency where overall impedance is mostly dominated by $R_{M}$ is applied to the sensor; the resultant current is then measured through a high-sensitivity readout circuit. Fig.3 shows the simplified circuit schematic of the overall readout electronics composed of a pulse generator, an analog interface, and a data acquisition (DAQ) board for digital signal processing (DSP).

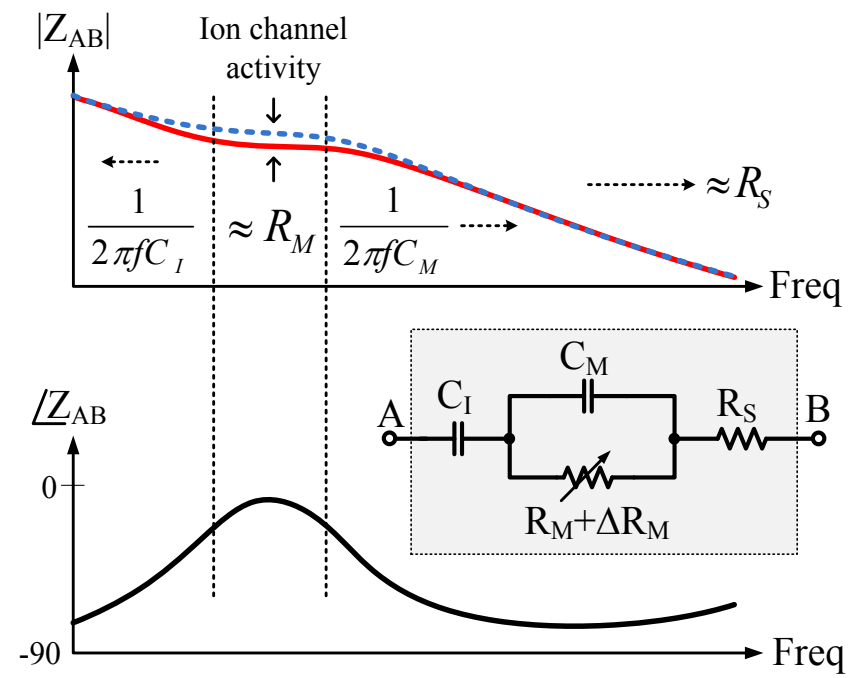

Figure 2. Typical impedance spectrum of a tethered lipid bilayer. The change in the impedance in response to ion channel activity is more significant at the frequencies where the membrane resistance is dominant.
A major problem of biosensors whose operation is based on resistance measurement is the fact that $\Delta R_{M}$ is generally much smaller than the nominal value of the $\mathrm{R}_{\mathrm{M}}$. This leads a relatively large nonspecific output current creating an output offset that may possibly saturate readout electronics even in the absence of a biological activity. In order to suppress the output offset by minimizing the nonspecific component of the resultant current, a pseudo-differential operation scheme is employed. In this approach, an external reference electrode having an impedance spectrum very close to that of the actual sensor is connected to the readout circuit. By applying differential voltage pulses, only the portion of the current related to ion channel activity is sensed by the interface electronics. Therefore, much higher sensitivities in the full supply range are attained. Although the mismatch between the sensing and the reference impedances is still an issue for reference circuits implemented with external R-C components, it is expected that any possible mismatch can be minimized by using reference electrode sites. These can be fabricated with the same process steps as the sensing electrodes and can be located as close as possible to the sensing ones within the array.

The readout electronics must be capable to detect very small levels of sensor output current. The most common and simplest way of measuring small currents is to use transresistance amplifiers. However, in standard CMOS processes it is not feasible to implement real resistors having very large values required for high-sensitive I-V conversion. Employing MOS transistors operating in subthreshold region can be an alternative solution; nevertheless, the channel resistances of such transistors are not well controlled due to process variations. Thus, an SC current integrator, which is

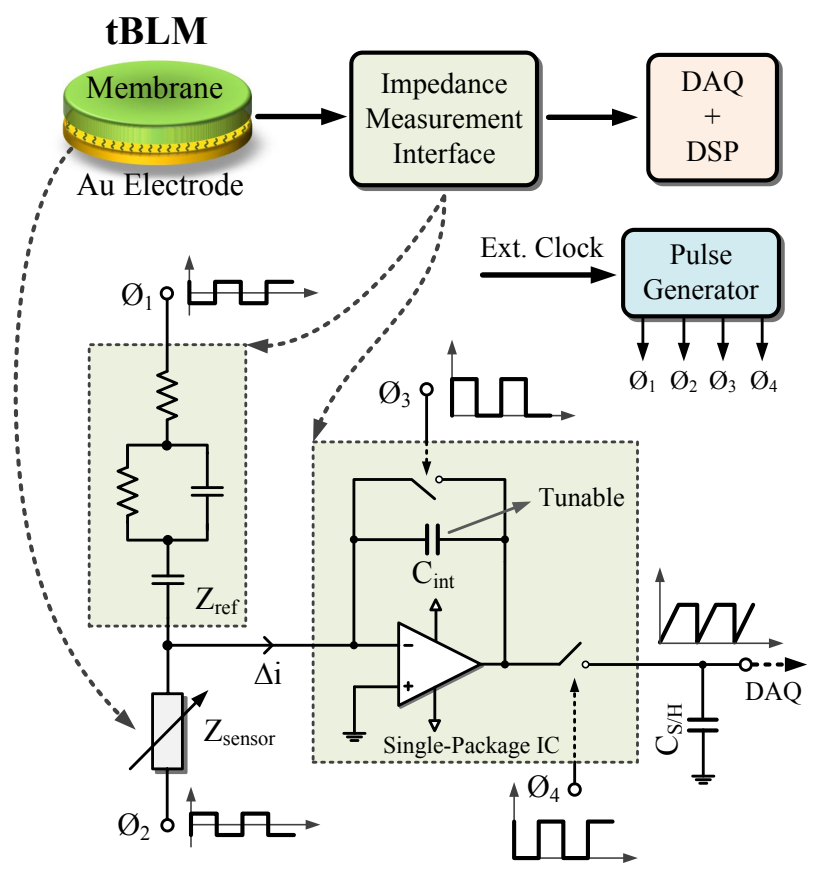

Figure 3. Simplified circuit schematic of the proposed switched-capacitor current integrator based impedance measurement circuit. 
widely used for MEMS capacitive sensors [7], is employed in our system. The circuit can easily be implemented on-chip and it provides high-sensitivity operation. The operation principle is as follows: in the reset phase, the unity-gain feedback provides a robust DC biasing at the sensing node, eliminating the effects of parasitics; then, in the integration phase, generated current in response to applied pulse is converted to voltage with a sufficiently high I-V conversion factor owing to very-large effective impedance of the integration capacitance $\left(\mathrm{C}_{\mathrm{int}}\right)$ at the frequencies involved.

The output of the SC integrator is then applied to a sample-and-hold circuit whose output is acquired by a DAQ board. This signal is digitally low-pass filtered by LabVIEW software (National Instruments) to extract the baseband voltage proportional to the membrane resistance change.

\section{TEST RESULTS}

The measurement circuit is implemented with high performance discrete components to improve experimental flexibility and reliability during the verification and characterization tests. These tests are performed in two steps: first, the functionality of the circuit is verified by the electrical equivalent model of the biosensor; then, the circuit is connected to actual sensors and results are compared to those of lock-in amplifier measurements.

Fig. 4 shows the plot of average output voltage change measured for discrete values of $R_{M}$ ranging from $1 \mathrm{Mohm}$ to $1.1 \mathrm{Mohm}$, while other model parameters of reference and sensing impedances are kept constant. For the integration capacitance of $10 \mathrm{pF}$ and the excitation voltage of $40 \mathrm{mV}_{\mathrm{pp}}$ at $10 \mathrm{~Hz}$, output voltages up to $1.5 \mathrm{~V}$ are achievable for $100 \mathrm{k} \Omega$ of resistance change. From the same plot, the $\mathrm{R}^{2}$ nonlinearity is calculated as $0.3 \%$.

The effect of the excitation frequency on the response of the proposed circuit is measured and compared to SPICE simulations. $\mathrm{R}_{\mathrm{M}}$ is changed from $1 \mathrm{Mohm}$ to $1.1 \mathrm{Mohm}$ and the resultant output voltage difference is plotted. Fig.5 gives

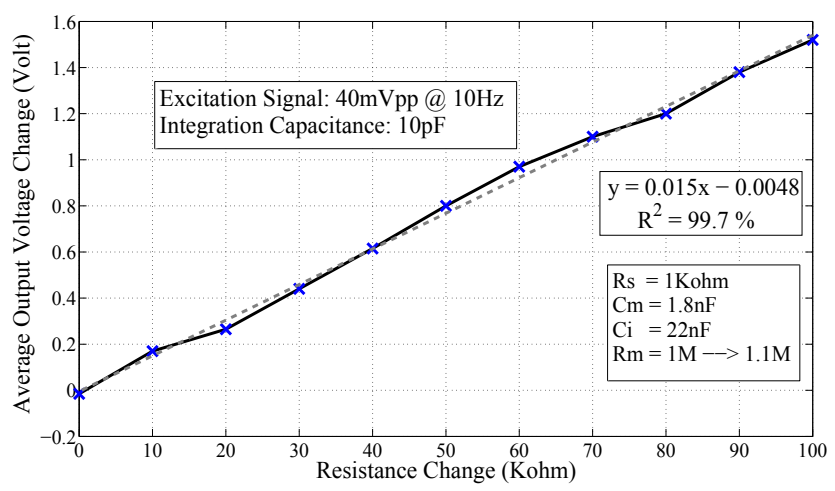

Figure 4. Resistance change vs. average output voltage change plot measured for discrete values of $\mathrm{R}_{\mathrm{M}}$ ranging from $1 \mathrm{Mohm}$ to $1.1 \mathrm{Mohm}$. the frequency response of the electrical equivalent model measured for set of frequencies ranging from $2 \mathrm{~Hz}$ to $200 \mathrm{~Hz}$. $\Delta \mathrm{R}_{\mathrm{M}}$ leads to higher sensitivities in a certain mid-frequency range as expected. Moreover, this plot also implies that measurement results are consistent with the simulations.

In order to compare the outputs of SC integrator and lock-in amplifier, the circuit response given in Fig.5 is converted to equivalent impedance change by using the sensitivity information derived from Fig.4. Fig.6 shows the magnitude of the impedance change measured by the proposed circuit and the lock-in amplifier. The distorted response of the lock-in amplifier is due to the limited number of data points since measuring with higher number of data points requires very long measurement durations at such low frequencies. The response of the SC circuit is in good agreement with the lock-in amplifier response except a shift in the frequency where the response reaches its peak value. Since the effective impedance of the integration capacitance is higher at lower frequencies, thus leading to higher sensitivities at lower frequencies, the overall frequency response shifts to the left as expected.

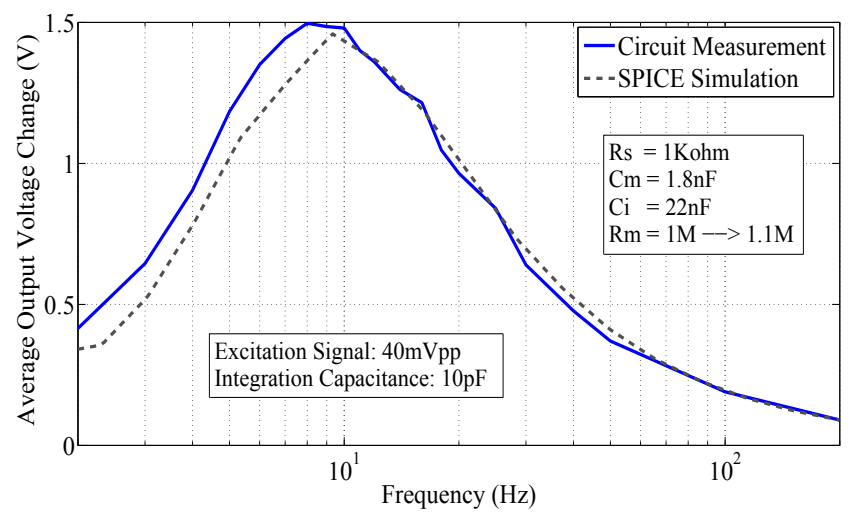

Figure 5. Measurement and simulation results for the average output voltage change when the membrane resistance is changed from $1 \mathrm{Mohm}$ to $1.1 \mathrm{Mohm}$ and excitation frequency is swept from $2 \mathrm{~Hz}$ to $200 \mathrm{~Hz}$.

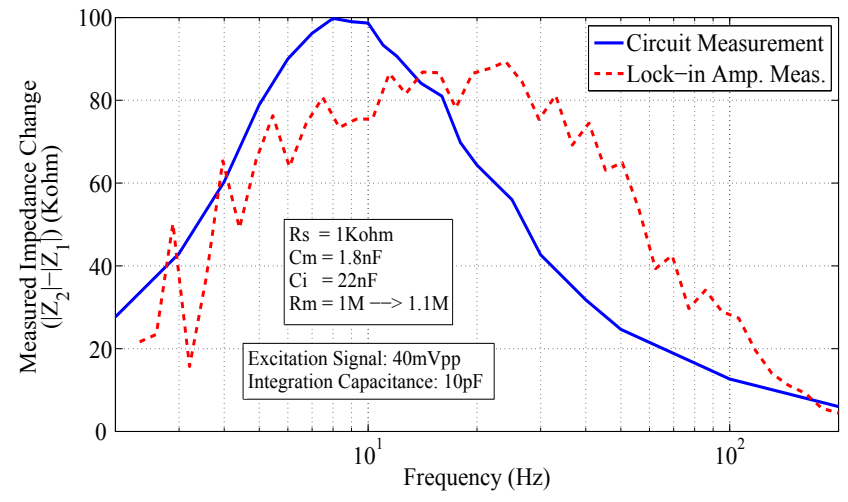

Figure 6. Frequency vs. impedance change plot measured with the proposed circuit and the lock-in amplifier when the membrane resistance is changed from $1 \mathrm{Mohm}$ to $1.1 \mathrm{Mohm}$. 
Having verified the circuit operation with electrical equivalent models, the proposed impedance measurement circuit is tested with the actual tBLM biosensors comprising $500 \mu \mathrm{m}$ diameter Au disk electrodes. The initial state of the membrane is first characterized by the lock-in amplifier. Fig.7 shows the real part of the measured impedance spectrum. From this plot, the nominal value of the $R_{M}$ is calculated as $100 \mathrm{Kohm}$. The sensor and the estimated reference impedance are then connected to the proposed impedance measurement circuit. Fig.8 shows the measured average output voltage after the addition of $0.3 \mu \mathrm{M}$ Gramicidin [6], an antibiotic creating ion channels in the membrane; thus, leading to a decrease in $\mathrm{R}_{\mathrm{M}}$. It is clearly seen that the ion channel formation and change in the membrane impedance can be monitored continuously through the real-time impedance measurement technique. DC offset due to the mismatch between reference and sensing impedances and low-frequency noise are the main problems observed; however, these can be solved by fabricating reference electrodes sites and employing circuits having $1 / \mathrm{f}$ noise cancellation techniques. The decrease in the membrane resistance is then verified by the lock-in amplifier, as shown in Fig.7.

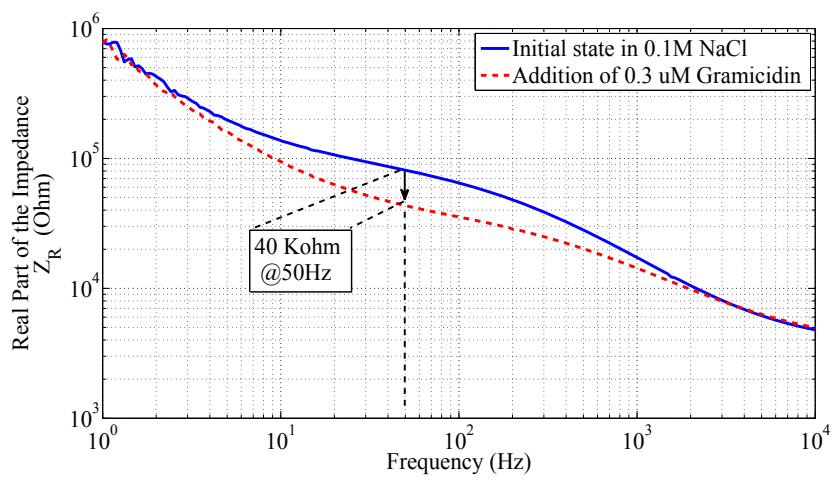

Figure 7. Real part of the impedance spectrum of a tethered lipid bilayer before and after the addition of $0.3 \mu \mathrm{M}$ Gramicidin, measured by the lock-in amplifier.

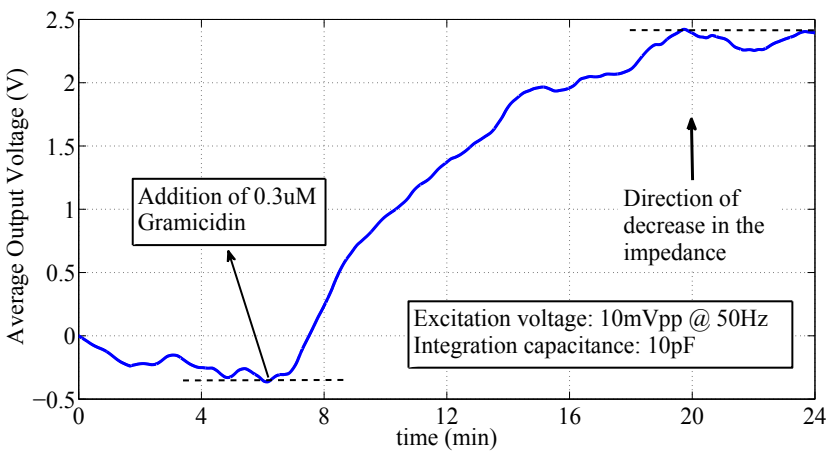

Figure 8. Measured average output voltage of the circuit after the addition of $0.3 \mu \mathrm{M}$ Gramicidin, when the sensor and the reference impedances are excited with differential square pulses having peak-to-peak amplitude of $10 \mathrm{mV}$ at $50 \mathrm{~Hz}$.
In addition, the system behavior is also tested under the influence of complex physiological fluids, such as blood serum or ascitic fluid. The increase of $\mathrm{R}_{\mathrm{M}}$ due to ligand binding is often masked in complex media by unspecific effects such as adsorption of proteins to local defects or partial damage of the lipid bilayer. However, consistent results between the SC integrator and the lock-in amplifier are invariably obtained.

\section{CONCLUSION}

A real-time, highly-sensitive and integrable impedance measurement technique for monitoring the membrane resistance change of tBLM biosensors in response to ion channel activity is presented. It is aimed to replace conventional electrochemical impedance spectroscopy techniques with compact and dedicated electronics enabling the realization of miniaturized lab-on-a-chip systems composed of high density biosensor arrays. In the proposed approach, the DC offset due to the nonspecific component of the generated current is minimized through pseudo-differential operation, and then the output current related to the biological activity is converted to voltage by a high-sensitivity SC integrator circuit. The circuit functionality was first tested with the electrical equivalent models of the sensor. Then, it was tested using tBLM biosensors by continuously monitoring ion channel formation. It was demonstrated that the SC circuit can provide real-time impedance measurements in good agreement with conventional impedance spectroscopy techniques. We concluded that the fully-integrated version of the proposed approach is a promising solution for tBLM sensor arrays having sensing and reference membranes on the same support.

\section{REFERENCES}

[1] S. Terrettaz and H. Vogel, "Investigating the function of ion channels in tethered lipid membranes by impedance," MRS Bulletin, Vol. 30, pp.207-210, 2005.

[2] A. Janshoff and C. Steinem, "Transport across artificial membranesan analytical perspective," Analytical and Bioanalytical Chemistry, Vol. 385, pp.433-451, 2006

[3] S. Heyse, T. Stora, E. Schmid, J. H. Lakey, and H. Vogel, "Emerging techniques for investigating molecular interactions at lipid membranes," Biochimica et Biophysica Acta, Vol. 85507, pp319-338, 1998.

[4] S. Terrettaz, W. P. Ulrich, R. Guerrini, A. Verdini, and H. Vogel, "Immunosensing by a synthetic ligand-gated ion channel," Angewandte Chem. Int. Edition, Vol. 40, pp.1740-1743, 2001.

[5] S.Terrettaz, M. Mayer, and H. Vogel, "Highly electrically insulating tethered lipid bilayers for probing the function of ion channels", Langmuir, Vol. 19, pp.5567-5569, 2003.

[6] B. A. Cornell, V. L. B. Braach-Maksvytis, L. G. King, P. D. J. Osman, B. Raguse, L. Wieczorek, and R. J. Pace, "A biosensor that uses ion-channel switches," Nature, Vol. 387, pp.580-583, June 1997.

[7] N. Yazdi, H. Kulah, and K. Najafi, "Precision Readout Circuits for Capacitive Microaccelerometers", Proc. of IEEE Sensors, Vol. 1, pp.28-31, Oct. 2004. 